\title{
Advances on the structure of the R2TP/Prefoldin-like complex
}

Hugo Muñoz-Hernández ${ }^{1 \S}$, Mohinder $\mathrm{Pal}^{2 \S}$, Carlos F. Rodríguez ${ }^{1,}$, Chrisostomos Prodromou $^{2}$, Laurence H. Pearl ${ }^{2}$ and Oscar Llorca ${ }^{1 *}$

1) Spanish National Cancer Research Centre (CNIO), Melchor Fernández Almagro 3, 28029 Madrid, Spain

2) Genome Damage and Stability Centre, School of Life Sciences, University of Sussex, Falmer, Brighton, UK

$\S$ These authors made equal contributions

* Correspondence and requests for materials should be addressed to O.L. (email: ollorca@cnio.es) 


\begin{abstract}
Cellular stability, assembly and activation of a growing list of macromolecular complexes require the action of HSP90 working in concert with the R2TP/Prefoldinlike (R2TP/PFDL) co-chaperone. RNA polymerase II, snoRNPs and complexes of PI3kinase-like kinases, a family that includes the ATM, ATR, DNA-PKcs, TRAPP, SMG1 and mTOR proteins, are among the clients of the HSP90-R2TP system. Evidence links the R2TP/PFDL pathway with cancer, most likely because of the essential role in pathways commonly deregulated in cancer. R2TP forms the core of the co-cochaperone and orchestrates the recruitment of HSP90 and clients, whereas prefoldin and additional prefoldin-like proteins, including URI, associate with R2TP, but their function is still unclear. The mechanism by which R2TP/PFLD facilitates assembly and activation of such a variety of macromolecular complexes is poorly understood. Recent efforts in the structural characterization of R2TP have started to provide some mechanistic insights. We summarize recent structural findings, particularly how cryo-electron microscopy (cryo-EM) is contributing to our understanding of the architecture of the R2TP core complex. Structural differences discovered between yeast and human R2TP reveal unanticipated complexities of the metazoan R2TP complex, and opens new and interesting questions about how R2TP/PFLD works.
\end{abstract}

\title{
Keywords
}

R2TP, Prefoldin-like, HSP90 co-chaperone, cryo-EM 


\section{The R2TP/Prefoldin-like (R2TP/PFDL) complex}

The HSP90 molecular chaperone assists for the assembly, cellular stability and activation of an eclectic set of macromolecular complexes. Despite much research on the structure and molecular processes involved, a profound mechanistic understanding of how HSP90 accomplishes its functions is still lacking. HSP90 participates in such a large variety of processes in part thanks to the assistance of co-chaperones. Cochaperones regulate HSP90 ATPase activity and help recruit specific client proteins to the chaperone (1).

The R2TP/Prefoldin-like (R2TP/PFLD) complex is the most complex HSP90 cochaperone yet described $(2,3)$. R2TP forms a core assembly made of 4 different proteins (see next section), and this is essential for the assembly and stability of several macromolecular complexes, among these, small nucleolar ribonucleoproteins (snoRNPs) (4), RNA polymerase II (Pol II) (3) and the phosphatidylinositol-3-kinaselike kinases (PIKKs) (5-9). The list of large assemblies requiring the assistance of HSP90 and R2TP for activation and stability is growing, including recent additions such as U5 snoRNPs $(10,11)$ and MRE11 $(6,12)$.

In the cell, R2TP associates with additional subunits $(3,10)$. The best characterized are a collection of prefoldin and prefoldin-like proteins forming the so called prefoldin (PFLD) complex, whose exact function is unclear. In addition, a variety of adaptor proteins and complexes seem to participate in the recruitment of some HSP90 clients to the R2TP complex. It has been proposed that the PFLD module could be one of the adaptors involved in the R2TP-mediated assembly of RNA polymerase (3). Also, 
Wdr92/Monad associates to the RPAP3 subunit of R2TP, and recent evidence suggest that Wdr92/Monad could contribute, in association to R2TP, to the assembly of axonemal dynein (13). Most of the currently known complexes needing the action of R2TP/PFLD have been found deregulated in cancer. Thus, the growing evidence implicating components of the R2TP/PFLD complex with cancer is no surprise (14-20).

\section{Composition of R2TP/PFDL}

Human R2TP comprises several copies of the AAA+ ATPases RUVBL1 and RUVBL2 (Rvb1p, Rvb2p in yeast), interacting with RNA polymerase II associated protein 3, RPAP3 (Tah1p in yeast), and a Pih1 domain-containing protein 1, PIH1D1 (Pihlp in yeast) (Fig. 1). RUVBL1 and RUVBL2 assemble hetero-hexameric rings as well as dodecameric double-ring complexes that interact with RPAP3/Tah1p and PIH1D1/Pih1p $(2,5,10)$.

RPAP3, a protein initially identified and named by its association with RNA Pol II using protein affinity purification coupled to mass spectrometry (21), was subsequently ascribed as a component of R2TP $(3,22)$. RPAP3 contains two tandem $\mathrm{N}$-terminal TPR domains that bind the conserved MEEVD C-terminal tail peptide of $\operatorname{HSP} 90(5,23,24)$

(Figure 1). Yeast Tahlp is a much smaller protein, comprising only 1 TPR $(5,25)$, although it has been shown to be able to form homodimers (26), but the importance of this observation in the context of the R2TP complex remains unknown.

PIH1D1 mediates recruitment of some potential clients and adaptors to R2TP through an N-terminal phosphopeptide-binding PIH domain recognizing a specific motif produced by casein kinase 2 (CK2) phosphorylation $(5,6,9)$. The PIH N-terminal domain acts as a phospho-peptide binding module, whereas a CS-domain at the C- 
terminus interacts with Tah1p $(5,25,26)$. In yeast, Pihlp acts as the scaffold for the interaction with Tah1p and the Rvb1p-Rvb2p heterohexameric ring (27-29).

Intriguingly, R2TP-like complexes can be formed when RUVBL1 and RUVBL2 interact with dynein assembly factors (DNAAFs) containing TPR and PIH domains, and which would carry some of the functions of RPAP3 and PIH1D1 $(13,30)$. These R2TPlike complexes would participate in the regulation of dynein assembly, but evidence for their existence in vivo was up to now mostly indirect. Just recently, Maurizy et al. (2018) describe several R2TP-like complexes detected by a systematic interaction analysis of the RPAP3 protein (31). Interestingly, these authors also reveal the formation of several R2TP-like complexes made of PIH-like and RPAP3-like proteins.

In mammals, R2TP associates with proteins of the PFDL module. This comprises prefoldin and additional prefoldin-like proteins, URI, PFDN2, PFDN6, PDRG1 and UXT, which associate with Pol II subunits $(3,11)$. The interaction of the Pol II subunit RPB5 with URI (Unconventional prefoldin RPB5 Interactor) has been interpreted as indication that the prefoldin module would assist Pol II assembly as part of a R2TP/PFLD complex $(3,32)$. How this happens is still poorly understood.

\section{RUVBL1-RUVBL2, a platform for protein recruitment}

X-ray crystallography and cryo-EM have revealed the structural organization of RUVBL1/Rvb1p and RUVBL2/Rvb2p, two ATPases that are closely related (33-38). The ATPase core is made of domains I (DI) and III (DIII), interacting in the form of hetero-hexameric rings with alternating subunits. Domain II (DII) is flexibly attached and protrudes from the ring, and includes an oligonucleotide-binding (OB) domain, 
which has been shown to interact with several unrelated proteins in the context of distinct complexes.

Al least in humans $(35,39)$, yeast (33) and Chaeetomiun thermophilim (34), a thermophilic fungus, DII domains from two hexamers interact together via their DII domains to assemble a dodecameric complex. The functional significance of hexamers versus dodecamers is extensively debated. Although the role of these different structures has not yet been resolved, recent structural insights suggest that hexamers are likely the most common functional form of RUVBL1-RUVBL2, which plays a major role as a platform that scaffolds interaction with other proteins. Interestingly, in all cases where structures have been reported at sufficient resolution to map their components, it is the DII-face of hexameric RUVBL-rings and the DII domains themselves that direct the interaction with other proteins, whether the complex is yeast R2TP $(27,28)$ (Fig. 2A-C), INO80 (40, 41) (Fig. 2D) or human R2TP (39) (Fig. 3). In this model, hexamers would be the "active" form of RUVBL1-RUVBL2 complex, capable of interaction with other proteins, whereas the dodecameric complexes could represent a form not competent for binding and relatively less active in its ATPase activity. In support of this, Tah1p-Pih1p stimulates the ATPase activity of the hexameric form of Rvb1p-Rvb2p complex $(27,28)$. However, our recent structure of the human R2TP complex revealed, for the first time to our knowledge, that the ATPase side of the RUVBL-ring can also scaffold protein-protein interactions by interaction through RPAP3 (39), whose implications are discussed below.

\section{Structural understanding of client and HSP90 recruitment}


A combination of biochemical and structural studies has provided a structural understanding of the basic features defining how R2TP recruits HSP90 and some of its clients. In yeast, a TPR domain in Tah1p interacts with the conserved MEEVD tail at the C-terminus of Hsp90 (Fig. 2C) $(5,25,26,42,43)$. Tah1p (12 kDa) is substituted by RPAP3 (75 kDa) in metazoa, containing two TPR domains that can bind HSP90 (5).

An unstructured C-terminal extension in Tah1p binds to the C-terminal CS domain in Pih1p $(5,25)$. The Pih1p-Tah1p complex is recruited to a hexameric ring of Rvb1pRvb2p, making multiple contacts with several DII domains of Rvb1p and Rvb2p subunits (Fig 2B-C) $(27,28)$. At the N-terminus of Pih1p and the equivalent metazoan PIH1D1, a phosphopeptide-binding PIH domain recognizes a CK2-phosphorylation motif present in some clients, such as Mre11p, or adaptors such as Tel2p that participate in the recruitment of PIKKs to R2TP $(5,6)$.

Not much is known about how R2TP recruits Pol II and other clients that do not contain the consensus PIH-binding motif. It has been proposed that several adaptors might connect R2TP and specific clients $(2,10,11)$. Some of these potential adaptors contain a PIH-binding motif that could serve this function, but in many other cases, how R2TP adaptors and clients are brought together remains unknown.

\section{Cryo-EM to the rescue. Visualizing the architecture of R2TP}

The 3D architecture and stoichiometry of the components in the assembled R2TP complex has remained elusive and poorly understood, in part due to the difficulties associated with the crystallization of such a large complex. In addition, we now know 
that these complexes are quite flexible, and thus are challenging for structural characterization.

Recently, advances in cryo-electron microscope (cryo-EM) have permitted the structural characterization of a number of large complexes that have resisted crystallization for many years, the PIKK family of kinases being a good example, where the structure of all human PIKKs, except SMG1, have been reported at sub-nanometer resolution with in the last few years (44). Following this trend, the first cryo-EM structures of R2TP complexes, from yeast, were published in 2017 (27, 28), providing some light on the structural organization and stoichiometry of this complex.

The first surprise was that both Rivera-Calzada et al. (2017), and Tian et al. (2017) revealed a 3:3:1:1 stoichiometry for the Rvb1p:Rvb2p:Tah1p:Pih1p complex in yeast $(27,28)$. Hexamers of Rvb1p-Rvb2p utilize their DII domains as a platform to interact with a copy of the Tah1p-Pihlp complex (Fig. 2A-C). Consequently, Rvb1p-Rvb2p double rings (dodecamers) are incompatible with the assembly of yeast R2TP. Given that each Rvb1p-Rvb2p ring is made of 3 molecules of Rvb1p and 3 molecules of Rvb2p, an understandable speculation was that up to 3 Tah1p-Pih1p complexes could bind each Rvb1p-Rvb2p ring. This would imply that several client-Hsp90 complexes could be managed by each R2TP. On the contrary, both studies clearly defined that only one Tah1p-Pihlp complex is accommodated within the cage formed by the DII domains of each Rvb1p-Rvb2p hexamer. Such stoichiometry has important implications in how yeast R2TP organizes clients and Hsp90, with each R2TP complex bringing in proximity one Hsp90 and one client, and avoiding the organization of super complexes containing several Hsp90 and clients per Rvb1p-Rvb2p ring. 
Interestingly, Tah1p-Pih1p binding to Rvb1p-Rvb2p induces conformational changes in the DII domains that stimulate ATP hydrolysis, but the relevance and consequences of this in R2TP function remains to be elucidated. Tian et al. (2017) suggested that ATP/ADP contributes to R2TP disassembly, based on the preference for Rvb1p-Rvb2p top views versus side views in the electron microscope when R2TP is incubated with ATP (27). Nonetheless, this electron microscopy evidence is weak since it is well documented that nucleotide binding to $\mathrm{AAA}+$ proteins can alter the preference of views in negative stain electron microscopy, and top views cannot be ascribed to either Rvb1p-Rvb2p or R2TP (27).

\section{Human R2TP, a flexible structure evolved to cope with a diversity of clients}

Recent cryo-EM of human R2TP revealed five remarkable features setting apart the much more elaborate architecture of human R2TP from the yeast complex (Fig. 3) (39).

(a) Whereas Pih1p scaffolds the interaction of all other R2TP components in yeast, this function is taken over by RPAP3 in human R2TP. RPAP3, the HSP90 binding TPR domain-containing component of R2TP, scaffolds the binding of RUVBL1-RUVBL2 and PIH1D1. Although cryo-EM suggests that PIH1D1 interacts with RUVBL1RUVBL2 (39), as in yeast R2TP $(27,28)$, interaction experiments clearly showed that PIH1D1 does not bind RUVBL1-RUVBL2 when RPAP3 C-terminal domain is not present (see next paragraph) (39).

(b) RPAP3 is a much larger protein than yeast Tah1p, and cryo-EM reveals that an alpha-helical C-terminal domain in RPAP3 engages at the ATPase face of the RUVBL 
ring (Fig. 2A-C), an interaction that is essential in holding the R2TP complex together. Interaction with the ATPase domains, rather than the DII face of the RUVBL ring, opens the possibility that other proteins could use a similar face/strategy to anchor to the RUVBL ring without the intervention of the DII domains. In this context, an NMR structure of the RPAP3 C-terminal domain has been also recently reported (Fig. 3D) (31), and the authors showed that two RPAP3-like proteins, CCDC103 and SPAG1, containing this domain, engage in the organization of R2TP-like complexes.

(c) RPAP3 spans both faces of the RUVBL ring (Fig. 3A), with the N-terminal TPRcontaining regions in RPAP3, which are involved in HSP90 recruitment, locating to the DII face of the RUVBL ring where the PIH domain client-recruitment component maps. This would allow easy access of HSP90 to its clients.

(d) The N-terminal HSP90 binding regions in RPAP3 are flexible and it can be envisioned that such architecture would facilitate the access of HSP90 to a large variety of complex clients that are recruited to R2TP. Perhaps, one of the roles of RPAP3 Cterminal domain is providing a tight anchor of RPAP3 to the RUVBL ring, freeing the N-terminal TPR-HSP90 containing region. However, it is noted that in the yeast R2TP complex flexibility appears to be limited to the residues linking the TPR-binding MEEVD motif to the C-terminal domain of HSP90 (Fig. 2C). In any case, the adaptability of the RUVBL1-RUVBL2 complex could be further enhanced by the recruitment of clients that are structurally diverse by a collection of adaptors and apparently different mechanisms.

(e) Each RUVBL ring can accommodate up to 3 RPAP3 molecules. The stoichiometry in vivo is unknown, but, since the R2TP core interacts with an ample number of 
adaptors and proteins to facilitate assembly and stability of different complexes, a likely scenario would be that the number of RPAP3 molecules bound to each RUVBL ring will depend on the context of those other interactions. The interaction with other proteins and adaptors might only happen with a single RPAP3, so that there is free available space for other essential proteins to interact with the RUVBL ring. Alternatively, there might be occasions when the presence of more than one RPAP3 per R2TP complex might have functional relevance. These complexes could bring several HSP90 and several clients in proximity. At least for the assembly of Pol II, the existence of several assembly intermediate complexes has been described, which consist of different protein-subunits that need to be brought together for Pol II assembly.

Together, the recent findings on the structure of the yeast and human R2TP core have revealed an elaborate structure that promotes the proximity between client and HSP90, and that inherent flexibility and adaptability allows such complexes to cope with diverse clients especially in the metazoan system. RPAP3 may play a major role in this flexibility, by providing a key scaffold function for other components of RUVBL1RUVBL2 complex. This key role of RPAP3 is underpinned by its C-terminal domain, whose function is to provide an anchor to the RUVBL ring.

\section{Conclusions and future perspectives}

The last two years have provided a significant advance in our structural and mechanistic understanding of the R2TP complex thanks to a combined effort of many groups and technologies. Cryo-EM has made an important contribution in providing a first 
structural view of the full R2TP complex. This year, cryo-EM has also revealed the structure of other RUVBL-containing complexes, which is starting to build a view of these ATPases as flexible scaffolds of a variety of protein-protein interactions.

Many questions remain open, and among them, is how URI, the prefoldin complex and other adaptors such as the TTT complex, associate to the R2TP core, and what this interaction means in both structural and functionally terms. It can be anticipated that the conformation of R2TP might be altered by its association with prefoldin and/or other of the several adaptors complexes that have been described. It can be expected that cryoEM will be a key technology in resolving such structures of these larger R2TP assemblies.

The cryo-EM structure of human R2TP, supported by the NMR structure of RPAP3 Cterminal domain, unexpectedly revealed that the ATPase face of the RUVBL is also utilized for protein-protein interactions. This opens the possibility that other proteins could also engage in this region, and additionally, that other regions in RUVBL could also function in protein binding.

The R2TP/PFDL-HSP90 complex contains several ATP binding sites, which are required for assistance in client assembly, activation and stability. Exactly, how and why this works, in mechanistic terms, is completely unknown. Available evidence in yeast suggest that the interaction of Pih1p and Tah1p with Rvb1p/Rvb2p stimulates their ATPase activity (28), while inhibiting the ATPase activity of HSP90 (45), but a clear understanding of what this implies in mechanistic terms, is missing. The scenario is even less clear in the case of human R2TP, and this is an area that will need to be investigated in order understand how R2TP/PFDL functions. 
In summary, the last few years have significantly increased our structural and mechanistic understanding of how the R2TP/PFDL complex works. Despite these advances, the ultimate question of how R2TP/PFDL works together with HSP90 to assemble, stabilize and activate a variety of client complexes remains essentially unsolved. Further research from multiple perspectives and a collection of methods, covering from the molecular to the cellular and organism level will be required. 


\section{References}

1. Prodromou C. The 'active life' of Hsp90 complexes. Biochim Biophys Acta. 2012;1823(3):614-23.

2. Vaughan CK. Hsp90 picks PIKKs via R2TP and Tel2. Structure. 2014;22(6):799-800.

3. Boulon S, Pradet-Balade B, Verheggen C, Molle D, Boireau S, Georgieva M, et al. HSP90 and its R2TP/Prefoldin-like cochaperone are involved in the cytoplasmic assembly of RNA polymerase II. Mol Cell. 2010;39(6):912-24.

4. Machado-Pinilla R, Liger D, Leulliot N, Meier UT. Mechanism of the AAA+ ATPases pontin and reptin in the biogenesis of H/ACA RNPs. RNA. 2012;18(10):183345.

5. Pal M, Morgan M, Phelps SE, Roe SM, Parry-Morris S, Downs JA, et al. Structural basis for phosphorylation-dependent recruitment of Tel2 to Hsp90 by Pih1. Structure. 2014;22(6):805-18.

6. Horejsi Z, Stach L, Flower TG, Joshi D, Flynn H, Skehel JM, et al. Phosphorylation-dependent PIH1D1 interactions define substrate specificity of the R2TP cochaperone complex. Cell Rep. 2014;7(1):19-26.

7. Kim SG, Hoffman GR, Poulogiannis G, Buel GR, Jang YJ, Lee KW, et al. Metabolic stress controls mTORC1 lysosomal localization and dimerization by regulating the TTT-RUVBL1/2 complex. Mol Cell. 2013;49(1):172-85.

8. Takai H, Xie Y, de Lange T, Pavletich NP. Tel2 structure and function in the Hsp90-dependent maturation of mTOR and ATR complexes. Genes Dev. 2010;24(18):2019-30.

9. Horejsi Z, Takai H, Adelman CA, Collis SJ, Flynn H, Maslen S, et al. CK2 phospho-dependent binding of R2TP complex to TEL2 is essential for mTOR and SMG1 stability. Mol Cell. 2010;39(6):839-50.

10. Houry WA, Bertrand E, Coulombe B. The PAQosome, an R2TP-Based Chaperone for Quaternary Structure Formation. Trends Biochem Sci. 2018;43(1):4-9.

11. Cloutier P, Poitras C, Durand M, Hekmat O, Fiola-Masson E, Bouchard A, et al. R2TP/Prefoldin-like component RUVBL1/RUVBL2 directly interacts with ZNHIT2 to regulate assembly of U5 small nuclear ribonucleoprotein. Nat Commun. 2017;8:15615. 12. von Morgen P, Burdova K, Flower TG, O'Reilly NJ, Boulton SJ, Smerdon SJ, et al. MRE11 stability is regulated by CK2-dependent interaction with R2TP complex. Oncogene. 2017;36(34):4943-50.

13. Zur Lage P, Stefanopoulou P, Styczynska-Soczka K, Quinn N, Mali G, von Kriegsheim A, et al. Ciliary dynein motor preassembly is regulated by $\mathrm{Wdr} 92$ in association with HSP90 co-chaperone, R2TP. J Cell Biol. 2018.

14. Saeki M, Egusa H, Kamano Y, Kakihara Y, Houry WA, Yatani H, et al. Exosome-bound WD repeat protein Monad inhibits breast cancer cell invasion by degrading amphiregulin mRNA. PLoS One. 2013;8(7):e67326.

15. Kakihara Y, Saeki M. The R2TP chaperone complex: its involvement in snoRNP assembly and tumorigenesis. Biomol Concepts. 2014;5(6):513-20. 
16. Huber O, Menard L, Haurie V, Nicou A, Taras D, Rosenbaum J. Pontin and reptin, two related ATPases with multiple roles in cancer. Cancer Res. 2008;68(17):6873-6.

17. Sang Y, Chen MY, Luo D, Zhang RH, Wang L, Li M, et al. TEL2 suppresses metastasis by down-regulating SERPINE1 in nasopharyngeal carcinoma. Oncotarget. 2015;6(30):29240-53.

18. Buren S, Gomes AL, Teijeiro A, Fawal MA, Yilmaz M, Tummala KS, et al. Regulation of OGT by URI in Response to Glucose Confers c-MYC-Dependent Survival Mechanisms. Cancer Cell. 2016;30(2):290-307.

19. Tummala KS, Gomes AL, Yilmaz M, Grana O, Bakiri L, Ruppen I, et al. Inhibition of de novo $\mathrm{NAD}(+)$ synthesis by oncogenic URI causes liver tumorigenesis through DNA damage. Cancer Cell. 2014;26(6):826-39.

20. Feng SW, Chen Y, Tsai WC, Chiou HC, Wu ST, Huang LC, et al. Overexpression of TELO2 decreases survival in human high-grade gliomas. Oncotarget. 2016;7(29):46056-66.

21. Jeronimo C, Forget D, Bouchard A, Li Q, Chua G, Poitras C, et al. Systematic analysis of the protein interaction network for the human transcription machinery reveals the identity of the 7SK capping enzyme. Mol Cell. 2007;27(2):262-74.

22. Cloutier P, Al-Khoury R, Lavallee-Adam M, Faubert D, Jiang H, Poitras C, et al. High-resolution mapping of the protein interaction network for the human transcription machinery and affinity purification of RNA polymerase II-associated complexes. Methods. 2009;48(4):381-6.

23. Kakihara Y, Houry WA. The R2TP complex: discovery and functions. Biochim Biophys Acta. 2012;1823(1):101-7.

24. Itsuki Y, Saeki M, Nakahara H, Egusa H, Irie Y, Terao Y, et al. Molecular cloning of novel Monad binding protein containing tetratricopeptide repeat domains. FEBS Lett. 2008;582(16):2365-70.

25. Jimenez B, Ugwu F, Zhao R, Orti L, Makhnevych T, Pineda-Lucena A, et al. Structure of minimal tetratricopeptide repeat domain protein Tah1 reveals mechanism of its interaction with Pih1 and Hsp90. The Journal of biological chemistry. 2012;287(8):5698-709.

26. Morgan RM, Pal M, Roe SM, Pearl LH, Prodromou C. Tah1 helix-swap dimerization prevents mixed Hsp90 co-chaperone complexes. Acta Crystallogr D Biol Crystallogr. 2015;71(Pt 5):1197-206.

27. Tian S, Yu G, He H, Zhao Y, Liu P, Marshall AG, et al. Pih1p-Tah1p Puts a Lid on Hexameric AAA+ ATPases Rvb1/2p. Structure. 2017;25(10):1519-29 e4.

28. Rivera-Calzada A, Pal M, Munoz-Hernandez H, Luque-Ortega JR, Gil-Carton D, Degliesposti G, et al. The Structure of the R2TP Complex Defines a Platform for Recruiting Diverse Client Proteins to the HSP90 Molecular Chaperone System. Structure. 2017;25(7):1145-52 e4.

29. Zhao R, Kakihara Y, Gribun A, Huen J, Yang G, Khanna M, et al. Molecular chaperone Hsp90 stabilizes Pih1/Nop17 to maintain R2TP complex activity that regulates snoRNA accumulation. J Cell Biol. 2008;180(3):563-78.

30. Olcese C, Patel MP, Shoemark A, Kiviluoto S, Legendre M, Williams HJ, et al. $\mathrm{X}$-linked primary ciliary dyskinesia due to mutations in the cytoplasmic axonemal dynein assembly factor PIH1D3. Nat Commun. 2017;8:14279. 
31. Maurizy C, Quinternet M, Abel Y, Verheggen C, Santo PE, Bourguet M, et al. The RPAP3-Cterminal domain identifies R2TP-like quaternary chaperones. Nat Commun. 2018;9(1):2093.

32. Mita P, Savas JN, Ha S, Djouder N, Yates JR, 3rd, Logan SK. Analysis of URI nuclear interaction with RPB5 and components of the R2TP/prefoldin-like complex. PLoS One. 2013;8(5):e63879.

33. Ewens CA, Su M, Zhao L, Nano N, Houry WA, Southworth DR. Architecture and Nucleotide-Dependent Conformational Changes of the Rvb1-Rvb2 AAA+ Complex Revealed by Cryoelectron Microscopy. Structure. 2016;24(5):657-66.

34. Lakomek K, Stoehr G, Tosi A, Schmailzl M, Hopfner KP. Structural basis for dodecameric assembly states and conformational plasticity of the full-length AAA+ ATPases Rvb1 . Rvb2. Structure. 2015;23(3):483-95.

35. Lopez-Perrote A, Munoz-Hernandez H, Gil D, Llorca O. Conformational transitions regulate the exposure of a DNA-binding domain in the RuvBL1-RuvBL2 complex. Nucleic Acids Res. 2012;40(21):11086-99.

36. Gorynia S, Bandeiras TM, Pinho FG, McVey CE, Vonrhein C, Round A, et al. Structural and functional insights into a dodecameric molecular machine - the RuvBL1/RuvBL2 complex. J Struct Biol. 2011;176(3):279-91.

37. Cheung KL, Huen J, Houry WA, Ortega J. Comparison of the multiple oligomeric structures observed for the Rvb1 and Rvb2 proteins. Biochem Cell Biol. 2010;88(1):77-88.

38. Torreira E, Jha S, Lopez-Blanco JR, Arias-Palomo E, Chacon P, Canas C, et al. Architecture of the pontin/reptin complex, essential in the assembly of several macromolecular complexes. Structure. 2008;16(10):1511-20.

39. Martino F, Pal M, Munoz-Hernandez H, Rodriguez CF, Nunez-Ramirez R, GilCarton D, et al. RPAP3 provides a flexible scaffold for coupling HSP90 to the human R2TP co-chaperone complex. Nat Commun. 2018;9(1):1501.

40. Eustermann S, Schall K, Kostrewa D, Lakomek K, Strauss M, Moldt M, et al. Structural basis for ATP-dependent chromatin remodelling by the INO80 complex. Nature. 2018;556(7701):386-90.

41. Ayala R, Willhoft O, Aramayo RJ, Wilkinson M, McCormack EA, Ocloo L, et al. Structure and regulation of the human INO80-nucleosome complex. Nature. 2018;556(7701):391-5.

42. Millson SH, Vaughan CK, Zhai C, Ali MM, Panaretou B, Piper PW, et al. Chaperone ligand-discrimination by the TPR-domain protein Tah1. Biochem J. 2008;413(2):261-8.

43. Back R, Dominguez C, Rothe B, Bobo C, Beaufils C, Morera S, et al. Highresolution structural analysis shows how Tah1 tethers Hsp90 to the R2TP complex. Structure. 2013;21(10):1834-47.

44. Imseng $\mathrm{S}$, Aylett $\mathrm{CH}$, Maier $\mathrm{T}$. Architecture and activation of phosphatidylinositol 3-kinase related kinases. Curr Opin Struct Biol. 2018;49:177-89.

45. Eckert K, Saliou JM, Monlezun L, Vigouroux A, Atmane N, Caillat C, et al. The Pih1-Tah1 cochaperone complex inhibits Hsp90 molecular chaperone ATPase activity. The Journal of biological chemistry. 2010;285(41):31304-12. 


\section{Acknowledgements}

This work was supported by the Project EXCELENCIA SAF2017-82632-P, AEIMINECO and cofounded by the European Regional Development fund (ERDF-EU) to OL and BES-2015-071348 to CFR. We thank Lidia Cerdán (CNB-CSIC) for help in Figure 4.

\section{Competing financial interests}

The authors declare no competing financial interests 


\section{Figure legends}

Figure 1. Components of the R2TP complex.

(A) Components of the yeast R2TP, where Pihlp plays a central scaffolding role. Domains are indicated using different colors, and blue arrows are used to indicate interactions between the proteins in R2TP. Numbers represent amino acid residues. DII domain inserts within the DI domain, and the denomination of "insertion" domain is sometimes used for this domain. For simplicity, we DI and DIII domains are not labeled.

(B) Components of the human R2TP, where RPAP3 scaffolds the interaction with PIH1D1 and RUVBL1-RUVBL2. HSP90 and PIH1D1 interacting regions in RPAP3 are indicated with blue arrows. Domains are indicated using different colors, and blue arrows highlight interactions of RPAP3 with PIH1D1 and RUVBL2. Numbers represent amino acid residues. DII domain inserts within the DI domain, and DI and DIII domains are not indicated.

Figure 2. Cryo-EM of yeast R2TP.

(A) Representative 2D average of yeast R2TP obtained using negative stain electron microscopy (28), where domains and regions have been labeled.

(B) Top view of the cryo-EM structure of yeast R2TP (28). Due to the limited resolution of the Pih1p-Tah1p components in the complex, a possible arrangement was proposed by fitting available crystal structures of Pih1p and Tah1p domains into the low-resolution density of the cryo-EM map. Tahlp is colored in yellow, and Pihlp in red. Rvb1p-Rvb2p is colored in blue, with the DII domains in darker blue color. 
(C) Side view of the same complex shown in "b".

(D) Structure of the INO80 core complex from the fungus Chaetomium thermophilum, in complex with the nucleosome (40). In the complex, the DII domains of the Rvb1Rvb2 hetero-hexamer scaffold other subunits in the complex.

Figure 3. Cryo-EM of human R2TP.

(A) Representative 2D average of human R2TP (39), where domains and regions have been labeled.

(B) Top view of the cryo-EM structure of RUVBL1-RUVBL2 bound to the C-terminal domain of RPAP3, obtained from the cryo-EM images of R2TP, but focusing refinement in the non-flexible regions (39). One copy of RPAP3 C-terminal domain is bound to each of the RUVBL2 subunits in the RUVBL1-RUVBL2 ring. Color codes: RUVBL1 (orange); RUVBL2 (blue); RPAP3 (yellow).

(C) Side view of the same complex shown in "b". The RPAP3 C-terminal domain binds to the ATPase side of the RUVBL ring.

(D) Comparison of the model for the complex between RUVBL1-RUVBL2 and the Cterminal domain of RPAP3 obtained by cryo-EM (39), and the NMR structure of the RPAP3 domain (31).

Figure 4. Model of the structural architecture of yeast and human R2TP and the functional implications. 
A

Rvb1p AAA+ DII (insertion) $^{130} \quad$ AAA+



Yeast R2TP

B

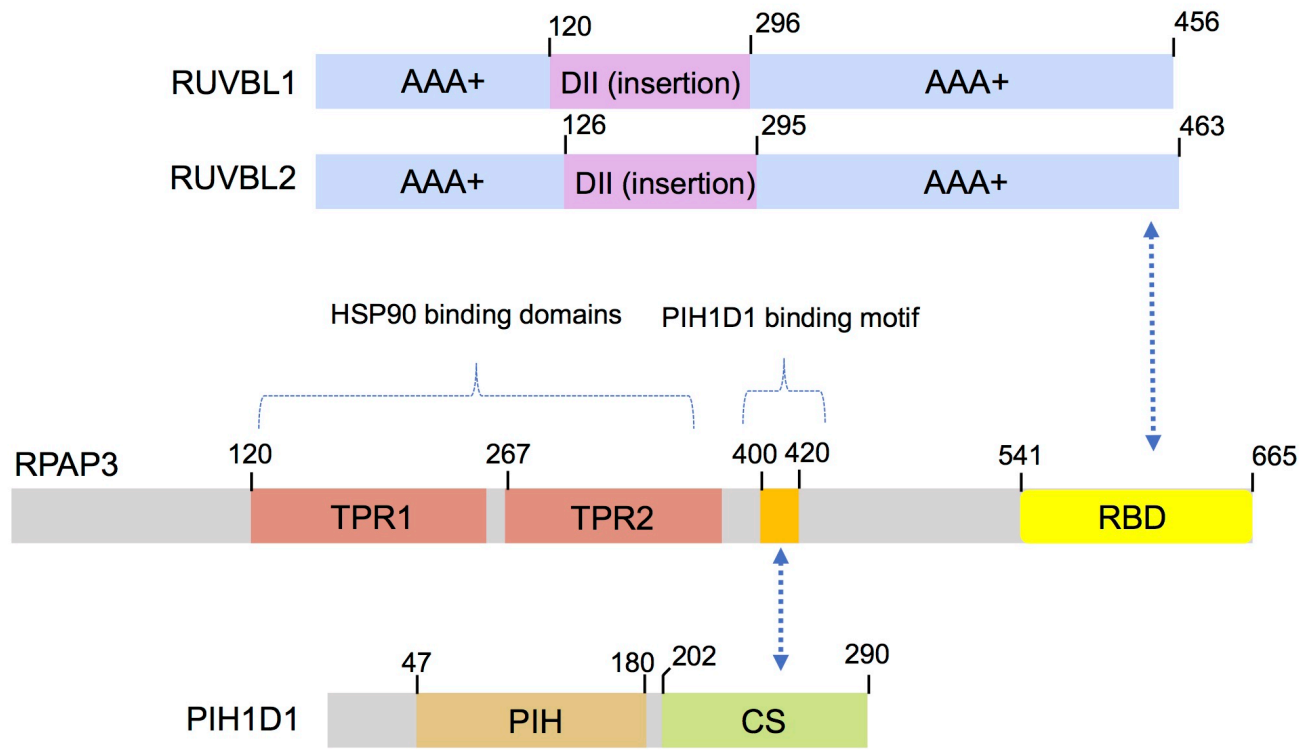

Human R2TP 
A

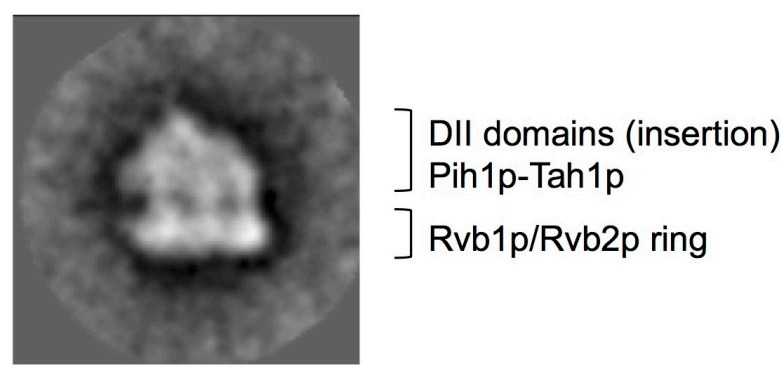

Negative stain 2D average of yeast R2TP

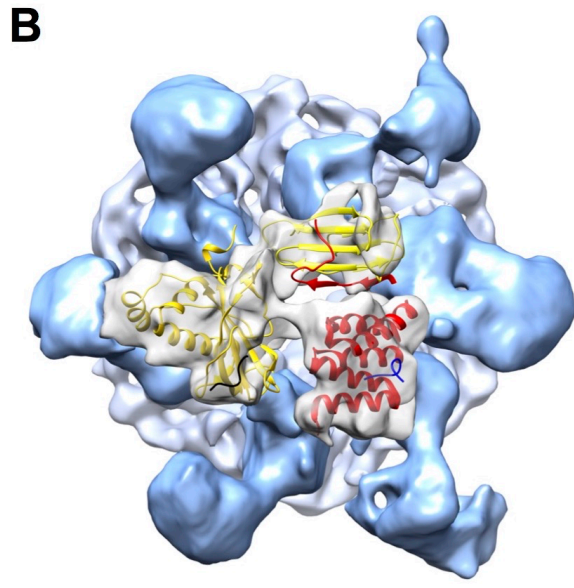

Top view of yeast R2TP
C

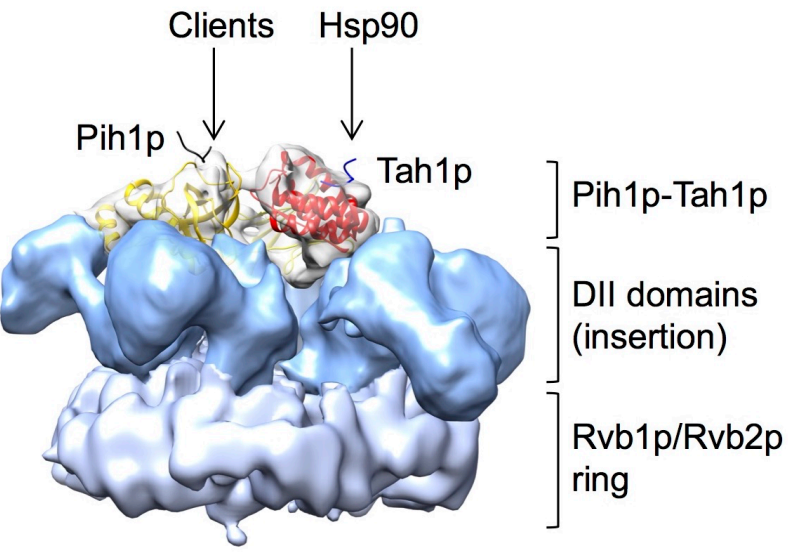

Side view of yeast R2TP

D



Side view of INO80-nucleosome complex (PDB 6fml) 
A



Flexible RPAP3 N-terminus

(HSP90 binding)

DII domains (insertion) and PIH1D1

RUVBL1-RUVBL2 ring

RPAP3 C-terminal domain

Cryo-EM 2D average of human R2TP

B

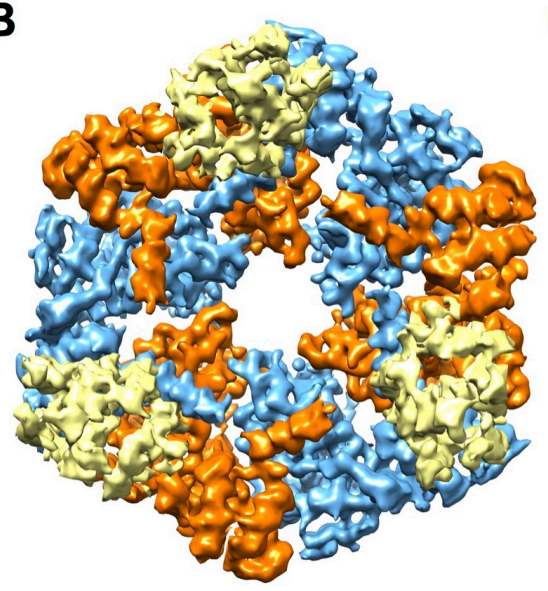

Top view of human R2TP

C

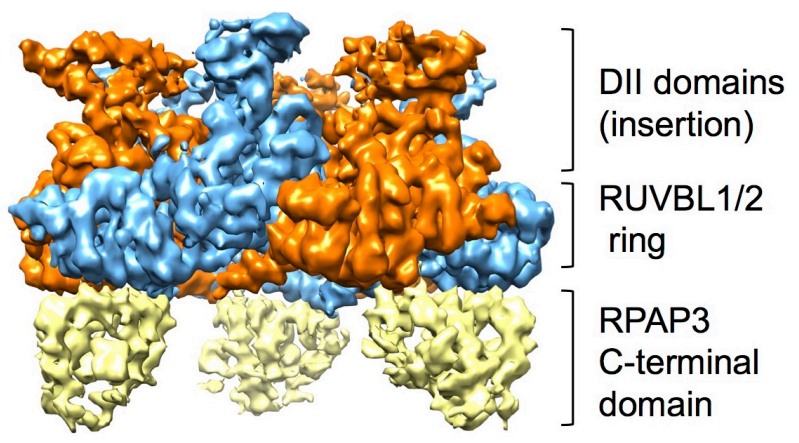

Side view of human R2TP

D




YEAST R2TP

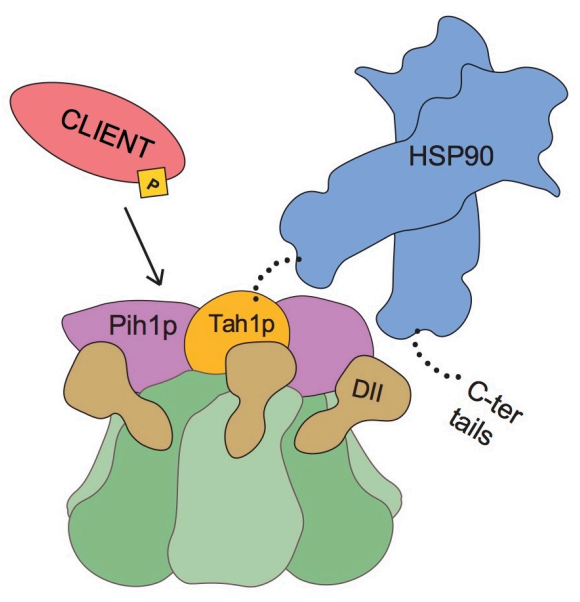



